OPEN ACCESS

Edited by:

Xi-Nian Zuo,

Chinese Academy of Sciences, China

Reviewed by:

Ting $X_{u}$,

Child Mind Institute, United States

Stavros I. Dimitriadis,

Cardiff University, United Kingdom

*Correspondence:

Peter Kochunov

pkochunov@mprc.umaryland.edu

†These authors have contributed equally to this work

Received: 05 July 2017 Accepted: 25 February 2019

Published: 12 March 2019

Citation:

Kochunov P, Patel B, Ganjgahi H, Donohue B, Ryan M, Hong EL,

Chen X, Adhikari B, Jahanshad N, Thompson PM, Van't Ent $D$, den

Braber A, de Geus EJC,

Brouwer RM, Boomsma DI, Hulshoff Pol HE, de Zubicaray Gl, McMahon KL, Martin NG, Wright MJ and Nichols TE (2019) Homogenizing Estimates of Heritability Among SOLAR-Eclipse, OpenMx, APACE, and FPHI Software Packages in

Neuroimaging Data.

Front. Neuroinform. 13:16. doi: 10.3389/fninf.2019.00016

\section{Homogenizing Estimates of Heritability Among SOLAR-Eclipse, OpenMx, APACE, and FPHI Software Packages in Neuroimaging Data}

\author{
Peter Kochunov ${ }^{1 * \dagger}$, Binish Patel ${ }^{1 \dagger}$, Habib Ganjgahi ${ }^{2 \dagger}$, Brian Donohue ${ }^{1}$, Meghann Ryan ${ }^{1}$, \\ Elliot L. Hong ${ }^{1 \dagger}$, Xu Chen ${ }^{3}$, Bhim Adhikari ${ }^{1}$, Neda Jahanshad ${ }^{4}$, Paul M. Thompson ${ }^{4}$, \\ Dennis Van't Ent ${ }^{5}$, Anouk den Braber ${ }^{5}$, Eco J. C. de Geus ${ }^{5}$, Rachel M. Brouwer ${ }^{5}$, \\ Dorret I. Boomsma ${ }^{5}$, Hilleke E. Hulshoff Pol ${ }^{6}$, Greig I. de Zubicaray ${ }^{7}$, Katie L. McMahon ${ }^{8}$, \\ Nicholas G. Martin ${ }^{9}$, Margaret J. Wright ${ }^{9,10}$ and Thomas E. Nichols ${ }^{11}$

\begin{abstract}
${ }^{1}$ Maryland Psychiatric Research Center, Department of Psychiatry, University of Maryland School of Medicine, Baltimore, MD, United States, ${ }^{2}$ Department of Statistics, University of Oxford, Oxford, United Kingdom, ${ }^{3}$ Department of Cognitive Center Rudolf Magnus, Department of Psychiatry, University Medical Center Utrecht, Utrecht, Netherlands, ${ }^{7}$ Faculty of Australia, ${ }^{8}$ Centre for Advanced Imaging, University of Queensland, Brisbane, QLD, Australia, ${ }^{9}$ QIMR Berghofer Medical Research Institute, Brisbane, QLD, Australia, ${ }^{10}$ Queensland Brain Institute, University of Queensland, Brisbane, QLD, Australia, ${ }^{11}$ Big Data Institute, University of Oxford, Oxford, United Kingdom
\end{abstract} \\ Neuroscience, Maastricht University, Maastricht, Netherlands, ${ }^{4}$ Imaging Genetics Center, Keck School of Medicine of USC, \\ Marina del Rey, CA, United States, ${ }^{5}$ Department of Biological Psychology, VU University, Amsterdam, Netherlands, ${ }^{6}$ Brain \\ Health, and Institute of Health and Biomedical Innovation, Queensland University of Technology (QUT), Brisbane, QLD,
}

Imaging genetic analyses use heritability calculations to measure the fraction of phenotypic variance attributable to additive genetic factors. We tested the agreement between heritability estimates provided by four methods that are used for heritability estimates in neuroimaging traits. SOLAR-Eclipse and OpenMx use iterative maximum likelihood estimation (MLE) methods. Accelerated Permutation inference for ACE (APACE) and fast permutation heritability inference (FPHI), employ fast, non-iterative approximation-based methods. We performed this evaluation in a simulated twin-sibling pedigree and phenotypes and in diffusion tensor imaging (DTI) data from three twin-sibling cohorts, the human connectome project $(\mathrm{HCP})$, netherlands twin register (NTR) and BrainSCALE projects provided as a part of the enhancing neuro imaging genetics analysis (ENIGMA) consortium. We observed that heritability estimate may differ depending on the underlying method and dataset. The heritability estimates from the two MLE approaches provided excellent agreement in both simulated and imaging data. The heritability estimates for two approximation approaches showed reduced heritability estimates in datasets with deviations from data normality. We propose a data homogenization approach (implemented in solar-eclipse; www.solar-eclipse-genetics.org) to improve the convergence of heritability estimates across different methods. The homogenization steps include consistent regression of any nuisance covariates and enforcing normality on the trait data using inverse Gaussian transformation. Under these conditions, 
the heritability estimates for simulated and DTI phenotypes produced converging heritability estimates regardless of the method. Thus, using these simple suggestions may help new heritability studies to provide outcomes that are comparable regardless of software package.

Keywords: DTI, heritability, imaging genetics, reproducability, genetics, population, computational methods

\section{INTRODUCTION}

Reproducibility is the cornerstone of scientific research. Recent reports on low reproducibility in biomedical research are raising concerns that have to be addressed within the scientific community (Ioannidis, 2014). The emerging field of imaging genetics is not immune to these challenges ${ }^{1}$. Imaging genetics applies modern statistical genetics methods to quantitative phenotypes extracted from high dimensional neuroimaging modalities and has to address replication challenges in both imaging and genetic domains (Thompson et al., 2010). Challenges in replication include low statistical power, complexity of analysis, large number of dependent variables, statistical complexity, and differences in the analysis approaches and software (Meyer-Lindenberg et al., 2008; Collins and Tabak, 2014). All these challenges apply to imaging genetics studies. Imaging genetic studies look for factors that typically explain a small proportion of variance $(<1 \%)$ and may require a large sample sizes $(N=1,000-100,000)$ to be statistically powerful (Thompson et al., 2014). Imaging genetic studies employ complex analyses involving both imaging and genetic specialized analysis software (Meyer-Lindenberg et al., 2008). We tested the agreement between heritability estimates provided by four methods that are used for heritability estimates in neuroimaging traits. We demonstrated that the heritability estimates may vary by method and sample and propose a way to homogenize the outcomes.

The incomplete description of methods and low statistical power are the two chief factors that are likely contributing to the lack of reproducibility in imaging genetics studies (Collins and Tabak, 2014). Imaging genetic studies combine methods from both imaging and genetic disciplines. These studies require software for extraction of imaging phenotypes and software for genetic analyses of imaging traits, each having individual operating characteristics. For example, the outputs of imaging and genetic software may differ between versions of the same analysis software and even with the same version of software on different operating systems (Gronenschild et al., 2012). Imaging genetic analyses may also suffer from low power because the contribution from common variations in genome to phenotypic variability is typically small $(\sim 0.1 \%)$, thus requiring large samples to achieve significance and obtain reproducible results (Flint and Munafò, 2013). This further underscores the need for a careful study of the potential biases among different software analysis tools. These methodological biases may lead to challenges to replicate imaging genetic findings if in-kind imaging or genetic software is used during replication.

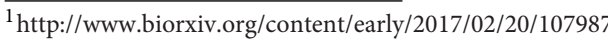

To address method-related biases, large consortia such as enhancing neuro imaging genetic meta analyses (ENIGMA) have developed standardized multi-site phenotype extraction and genetic analyses pipelines. In this manuscript, we consider the impact of analysis method for the estimation of heritability. We compared four approaches: two commonly used genetic analysis packages (SOLAR-Eclipse and OpenMx), and two recently developed accelerated heritability estimation methods [accelerated permutation inference for ACE (APACE), and fast permutation heritability inference (FPHI)]. These packages use the same variance component model and definition of heritability, but use different numerical methods and data preprocessing steps to calculate the proportion of variance attributed to additive genetic factors. We performed this study to (A) analyze if heritability estimates derived by the four packages' analyses are comparable to one another and; (B) develop a homogenization approach that minimizes the variability in heritability estimates across the four packages.

We performed these analyses using two datasets: a simulated-with known additive genetic contribution and an experimental-consisting of fractional anisotropy (FA) measurements collected in twins and siblings by three independent studies. FA is the most commonly analyzed scalar parameter extracted from diffusion tensor imaging (DTI; Basser et al., 1994; Basser and Pierpaoli, 1996) and is a sensitive index of fiber coherence, myelination levels, and axonal integrity (Thomason and Thompson, 2011). FA values are under a strong genetic control (Geng et al., 2012; Jahanshad et al., 2013; Shen et al., 2014). Individual differences in FA values are predictive of cognitive performance (Kochunov et al., 2016, 2017) and it is a promising phenotype for multiple neuropsychological disorder including schizophrenia (Friedman et al., 2008; PerezIglesias et al., 2010; Alba-Ferrara and de Erausquin, 2013; Kochunov et al., 2013; Mandl et al., 2013; Nazeri et al., 2013). All experimental data were processed using the harmonization protocol previously developed by ENIGMA and provided on-line at http://enigma.ini.usc.edu/ongoing/dti-working-group/). This included the use of the ENIGMA protocol for following the QA/QC steps for each site, registration to the ENIGMA-DTI target, extraction of white matter skeleton, followed by extraction of tract-average FA values.

\section{MATERIALS AND METHODS}

\section{Heritability Estimation Methods}

We evaluated the agreement in quantification of the Additive genetic and Environmental, AE, components of the phenotypic variance in simulated and imaging genetic datasets among 
four heritability calculation methods. SOLAR-Eclipse ${ }^{2}$ and Open $\mathrm{Mx}^{3}$ use the iterative maximum likelihood estimation (MLE) approach to fit quantitative genetics variance components models. The iterative MLE approach is used to determine the parameters that maximize the compatibility between the fitted model and the data. It is a versatile computational approach that produces estimates that are optimally precise asymptotically (Almasy and Blangero, 1998; Blangero et al., 2001). SOLAR-Eclipse is an extensive and flexible imaging genetics analysis software package. SOLAR-Eclipse functions include calculation of heritability, genetic correlation, linkage and genome-wide association analysis (Almasy and Blangero, 1998; Blangero et al., 2001). SOLAR-Eclipse polygenic function uses MLE to perform genetic analyses in the pedigrees of arbitrary size and complexity, including twin-siblings and complex multigenerational family designs. SOLAR-Eclipse is frequently used in imaging genetic studies especially in the multi-site analyses that aggregate measurements across multiple datasets using meta and mega-analyses (Jahanshad et al., 2013; Kochunov et al., 2014, 2015). OpenMx is an extensive and flexible structural equation modeling and path analysis library for [R] software (Boker et al., 2011). OpenMX is frequently used by imaging genetic studies to calculate heritability and genetic correlation in twin-siblings pedigrees (Jahanshad et al., 2010; Bootsman et al., 2016). Like SOLAR-Eclipse, OpenMx uses an iterative MLE method for calculation of heritability parameters.

APACE model and FPHI use statistical approximations to estimate heritability values. APACE uses a regression approach based on the squared differences of twin pairs, a variant of a U-statistic (Chen et al., 2013; Chen, 2014), while FPHI starts with the same likelihood as used in SOLAR-Eclipse but uses a single-step, rather than iterative, optimization (Ganjgahi et al., 2015). This overcomes the main limitation of the MLE-based software: long computational times. The iterative MLE heritability calculations in SOLAR-Eclipse and OpenMx can take $\sim 1$ s per trait in a pedigree of 1,000 subjects. Therefore, MLE-based heritability analyses require access to large computational clusters to perform imaging genetic analyses that involve $10^{4-} 10^{6}$ voxel-wise traits. The non-iterative estimates from APACE and FPHI offer appreciable $\left(\sim 10^{3}\right)$ gains in computational efficiency. This allows performing voxel-wise heritability analyses on a single workstation. While APACE is only intended for twin or twin-plus-sibling designs, FPHI can use any kinship structure, like SOLAR-Eclipse.

The four software packages were used to compare additive genetic contribution (heritability) in simulated and experimental data using twin family study designs. For experimental data we used DTI acquisitions from three different studies. The human connectome project (HCP; Van Essen et al., 2012), is a large-scale international collaboration aimed at elucidating the genetic and environmental sources of normal variability within the structural and functional connections of the human brain. The other two twin and sibling datasets were drawn

\footnotetext{
${ }^{2}$ www.solar-eclipse-genetics.org

${ }^{3}$ openmx.ssri.psu.edu
}

from the ENIGMA project, specifically from the ENIGMA-DTI workgroup whose focus is the analysis of DTI data. The first of these is the netherlands twin register (NTR) that collected DTI data in normally developing adolescent twins and siblings. And the other ENIGMA-DTI source is the Brain Structure and Cognition: an Adolescent Longitudinal Twin Study into Genetic Etiology (BrainSCALE). The BrainSCALE dataset collected DTI data in young adult twins and siblings. Subjects for NTR and BrainSCALE datasets were recruited from the same twin register in Netherlands.

We compare heritability estimates for tract-wise average FA values using ENIGMA-DTI, HCP, and simulated data. FA is a widely used quantitative measure of white matter microstructure (Basser et al., 1994; Basser and Pierpaoli, 1996) calculated from the diffusion tensor model of water diffusion (Thomason and Thompson, 2011). Studies suggest FA is an important biomarker in clinical studies, since it is a sensitive index of white matter integrity in Alzheimer's disease (Clerx et al., 2012; Teipel et al., 2012), general cognitive function (Penke et al., 2010a,b), and several neurological and psychiatric disorders (Sprooten et al., 2011; Barysheva et al., 2012; Carballedo et al., 2012; Kochunov et al., 2013; Mandl et al., 2013). Overall, our goal was to determine if additive genetic contribution (heritability) is comparable between software packages regardless of the variation in the twin-sibling cohort data. Our hypothesis was that estimates of heritability would be consistent amongst the cohorts, irrespective of the variability in cohort data and software package.

\section{Simulated Data}

A simulated $N=1,000$ person twin-sibling pedigree with 250 monozygotic (MZ) twins, 250 dizygotic (DZ) twins, and 500 founders (not included in the phenotype file) was created using SOLAR-Eclipse simulate function. SOLAR-Eclipse simulation functionality was also used to produce a data set of 10,000 traits with heritability estimates varied uniformly between 0 and $100 \%$. All simulated traits had normal distribution and did not include effects of covariates.

\section{Experimental Data}

\section{Human Connectome Project (HCP)}

- Subjects: the cohort contained 481 (194/287 M/F; average age $29.1 \pm 3.5$ ) healthy participants of the HCP for whom the scans and data were released in June 2014 (humanconnectome.org) after passing the HCP quality control and assurance standards (Marcus et al., 2013). The participants in the HCP study were recruited from the Missouri Family and Twin Registry, a large population-based study (Van Essen et al., 2012). This release included 117 twin pairs (57 MZ and $60 \mathrm{DZ}$ pairs), and 246 of their siblings. The full set of inclusion and exclusion criteria is detailed elsewhere (Van Essen et al., 2012).

- Imaging: diffusion data was collected at Washington University in St. Louis using a customized Siemens Magnetom Connectome 3-Tesla scanner with a $100 \mathrm{mT} / \mathrm{m}$ maximum gradient strength and a 32-channel head coil. Details on the scanner, image acquisition and reconstruction are provided 
in Ugurbil et al. (2013) $)^{4}$. Diffusion data were collected using a single-shot, single refocusing spin-echo, echo-planar imaging sequence with $1.25 \mathrm{~mm}$ isotropic spatial resolution $(\mathrm{TE} / \mathrm{TR}=89.5 / 5520 \mathrm{~ms}, \mathrm{FOV}=210 \times 180 \mathrm{~mm})$. Three gradient tables of 90 diffusion-weighted directions and six $b=0$ images each, were collected with right-to-left and left-to-right phase encoding polarities for each of the three diffusion weightings $\left(b=1,000,2,000\right.$, and $\left.3,000 \mathrm{~s} / \mathrm{mm}^{2}\right)$. The total imaging time for collection of diffusion data was approximately $1 \mathrm{~h}$.

\section{Netherlands Twin Register (NTR)}

- Subjects: the cohort consisted of 246 adults (93/153 M/F; average age $33.9 \pm 10.1$, range 19-57), recruited from the NTR and consisted of $72 \mathrm{MZ}$ pairs, $48 \mathrm{DZ}$ pairs, and six siblings. Exclusion criteria consisted of having any metal material in the head, having a pacemaker, a history of any major medical conditions or psychiatric illness (den Braber et al., 2008, 2011, 2012).

- Imaging: DTI data were collected on a 3-Tesla Philips Intera MR scanner (32 diffusion-weighted volumes with different non-collinear diffusion directions with $b$ factor $=1,000 \mathrm{~s} / \mathrm{mm}^{2}$ and one $b$-factor $=0 \mathrm{~s} / \mathrm{mm}^{2}$ image, flip angle $=90$ degrees; 38 axial slices of $3.0 \mathrm{~mm}$; no slice gap; voxel size, $2.0 \times 2.0 \times 3.0 \mathrm{~mm} ; \mathrm{FOV}=230 \mathrm{~mm}$; $\mathrm{TE}=94 \mathrm{~ms} ; \mathrm{TR}=4,863 \mathrm{~ms}$; no cardiac gating; and total scan duration $=185 \mathrm{~s})$.

\section{Brain Structure and Cognition: An Adolescent Longitudinal Twin Study into Genetic Etiology (BrainSCALE)}

- Subjects: the sample comprised of 199 children (100M/99F; average age $9.2 \pm 0.1$, range 9.0-9.6). It included $42 \mathrm{MZ}$ and $57 \mathrm{DZ}$ twin pairs that were recruited from families participating in the BrainSCALE cohort (van Soelen et al., 2012) that were recruited from the NTR (van Beijsterveldt et al., 2013). Exclusion criteria consisted of having any metal material in the head, having a pacemaker, a known history of any major medical condition or psychiatric illness. Zygosity was determined based on DNA polymorphisms, using 8-11 highly polymorphic di-, tri- and tetranucleotide genetic markers and confirmed by genome-wide single nucleotide polymorphism data.

- Imaging: DTI data were collected on a 1.5 Philips Achieva MR scanner (32 diffusion-weighted volumes with different non-collinear diffusion directions with $b$ factor $=1,000 \mathrm{~s} / \mathrm{mm}^{2}$ and eight diffusion-unweighted volumes with $b$-factor $=0 \mathrm{~s} / \mathrm{mm}^{2}$; parallel imaging SENSE factor $=2.5$; flip angle $=90$ degrees; 60 slices of $2.5 \mathrm{~mm}$; no slice gap; $96 \times 96$ acquisition matrix; reconstruction matrix $128 \times 128$; $\mathrm{FOV}=240 \mathrm{~mm} ; \mathrm{TE}=88 \mathrm{~ms} ; \mathrm{TR}=9,822 \mathrm{~ms}$; two repetitions; no cardiac gating; and total scan duration $=296 \mathrm{~s}$ ). More information may be found in Brouwer et al. (2010, 2012).

\footnotetext{
${ }^{4}$ https://www.humanconnectome.org/documentation/S500/HCP_S500_ Release_Reference_Manual.pdf
}

\section{ENIGMA-DTI Processing}

We used ENIGMA-DTI protocol to extract whole-brain and tract-wise average FA values for experimental datasets. These protocols are detailed elsewhere (Jahanshad et al., 2013) and are available online at http://enigma.ini.usc.edu/protocols/dtiprotocols/. In brief, FA images from all subjects were non-linearly registered to the ENIGMA-DTI target FA image using FSL's FNIRT (Smith et al., 2006). This target was created as a minimal deformation target based on images from the participating studies as previously described (Kochunov et al., 2002; Jahanshad et al., 2013). The data were then processed using FSL's tract-based spatial statistics (TBSS) analytic method (Smith et al., 2006) modified to project individual FA values onto the ENIGMA-DTI skeleton mask. After extracting the skeletonized white matter and the projection of individual FA values, ENIGMA tract-wise regions of interest (ROIs), derived from the Johns Hopkins University (JHU) white matter parcellation atlas available as a part of FSL, were transferred to extract the mean FA across the full skeleton and average FA values for major white matter tracts. The protocol, target brain, ENIGMA-DTI skeleton mask, source code and executables are all publicly available ${ }^{5}$. This protocol was shown to provide highly replicable measurements based on test-rest analyses in human subjects (Acheson et al., 2017; McGuire et al., 2017).

\section{Inverse Normal Transformation}

Multivariate quantitative trait models are sensitive to outliers, skewness, kurtosis and other deviations from normal distribution. Therefore, we consider the use of a rank-based inverse normal transformation to ensure the normal distribution in quantitative traits. For each phenotype, rank values are replaced with the expected ranked values of a standard normal distribution with the same number of observations. While it cannot ensure multivariate normality, it does ensure that each univariate distribution is normal and thus reduces the impact of outliers; for more discussion on this transformation see (Beasley et al., 2009). We implemented inverse normalization in SOLAR-Eclipse as the "polyclass_normalize" functions. This function produces inverse normalized residuals for the trait after regression of all covariates. The output from this function was used for the secondary analyses of the imaging data where we first analyze the raw data and then compare our results after the application of the inverse normal transformation to the residual data.

\section{Heritability Analysis}

Heritability analyses were performed in the simulated and FA traits. Heritability $\left(\mathrm{h}^{2}\right)$ is the proportion of the total phenotypic variance $\left(\sigma_{P}^{2}\right)$ that can be explained by the genetic effects of genes $\left(\sigma_{g}^{2}\right)$,

$$
\mathrm{h}^{2}=\mathrm{s}_{\mathrm{g}}^{2} / \mathrm{s}_{\mathrm{P}}^{2}
$$

\section{MLE Based Analysis}

SOLAR-Eclipse and OpenMX employ MLE based variance decomposition approach that is an extension of the strategy

\footnotetext{
$\overline{{ }^{5} \text { https://www.nitrc.org/projects/enigma_dti }}$
} 
developed by Amos (1994). The multivariate normal covariance matrix $\Omega$ for a pedigree of individuals is given by

$$
\Omega=2 \cdot \Phi \cdot \mathrm{s}_{\mathrm{g}}^{2}+\mathrm{I} \cdot \mathrm{s}_{\mathrm{e}}^{2}
$$

where $\Phi$ is the kinship matrix representing the pair-wise kinship coefficients among related individuals, $\sigma_{e}^{2}$ is the variance due to individual-specific environmental effects, and $I$ is an identity matrix (under the assumption that all environmental effects are uncorrelated among family members). Narrow sense heritability is defined as the fraction of phenotypic variance $\sigma_{P}^{2}$ attributable to additive genetic factors. In twin designs a third variance parameter is can be identified and may be added to the model, $\sigma_{c}^{2}$, for the common environment shared by twins and siblings growing up in the same family. This three-parameter model is known as the ACE model, while the two-parameter model (Equation 2) is referred to as the AE model.

The variance parameters are estimated by comparing the observed phenotypic covariance matrix with the covariance matrix predicted by kinship (Almasy and Blangero, 1998). Significance of heritability is tested by comparing the likelihood of the model in which $\sigma_{g}^{2}$ is constrained to zero with that of a model in which $\sigma_{g}^{2}$ is estimated. Twice the difference between the $\log _{e}$ likelihoods of these models yields a test statistic, which is asymptotically distributed as a 1/2:1/2 mixture of a $X^{2}$ variable with 1 degree-of-freedom and a point mass at zero.

\section{The Accelerated Permutation for the ACE Model (APACE)}

$\mathrm{APACE}^{6}$ uses an approximation technique developed originally for animal genetics studies (Grimes and Harvey, 1980) and is based on the result that squared differences of pair's of subjects' data reflect their covariance. Thus, the squared differences among the $\mathrm{DZ}, \mathrm{MZ}$ and unrelated subjects can be entered into a linear regression model to estimate the variance parameters (Grimes and Harvey, 1980). The speed advantage of APACE over MLE approaches allows a permutation analysis to compute familywise error corrected $P$-values for voxel-wise imaging measures.

\section{Fast Permutation Heritability Inference (FPHI)}

SOLAR-Eclipse's iterative MLE approach is accelerated by the use of a data transformation based on the eigenvectors of the kinship matrix $\Phi$ (Blangero et al., 2013). This transformation converts the dependent data from related subjects into data that is independent but has heterogeneous-variance. SOLAR-Eclipse uses this simplified model to obtain iterative MLE estimates using linear regressions. The FPHI approach uses the same likelihood and data transformation, but then performs just a single step estimation to produce an asymptotically unbiased estimate (Ganjgahi et al., 2015). The FPNI technique is implemented SOLAR-Eclipse as the CPU and graphics processing unit (GPU) functions. The CPU version of FPHI provides a significant $\left(10^{3}\right)$ computational acceleration relative to the iterative MLE estimation in SOLAR-Eclipse, while the graphics processing unit (GPU) version further improves this performance $\left(\sim 10^{6}\right)$ vs. iterative MLE approach.

\footnotetext{
$\overline{{ }^{6} \mathrm{http}: / / \text { warwick.ac.uk/tenichols/software/APACE }}$
}

All analyses with imaging data were conducted with age, sex, age $^{2}$, age $\times$ sex, and age ${ }^{2} \times$ sex included as covariates.

\section{RESULTS}

\section{Heritability Analyses-Simulated}

Figure 1 shows the scatter plots of four methods using a simulated dataset of heritability values distributed between 0 and 1. The two ML-based methods (SOLAR-Eclipse and OpenMX) showed an excellent agreement $(r=0.999$, slope $=1.000$, intercept $=0.000)$ with the expected heritability values and with each other (Figure 1). We quantified bias as estimated $h^{2}$ minus true $\mathrm{h}^{2}$ and "average spread" as the absolute bias divided by true value (i.e., |estimated - true|/true). In the simulated dataset, the two ML-based methods show zero bias (absolute value bias $<10^{-6}$ ) and the average spread in heritability estimates of $1.2 \%$. The APACE and FPHI methods showed excellent overall agreement with expected values (APACE: absolute value of bias $=10^{-5}, r=0.997$, slope $=0.997$, intercept $=0.005$; FPHI: absolute value of bias $=10^{-6}, r=0.998$, slope $=0.999$, intercept $=0.001)$. APACE showed significantly higher average spread than the FPHI method: 3.7 vs. $2.2 \%\left(p=10^{-10}\right)$.

\section{Heritability Analyses-Diffusion Data}

The heritability analyses were performed in FA data for 49 tracts in HCP, NTR and BrainScale cohorts using age, sex, age $^{2}$, age $\times$ sex, and age ${ }^{2} \times$ sex as covariates. The two ML-based method showed excellent agreement in all three datasets (Figure 2). The best agreement was observed in BrainScale data $(r=0.99$, slope $=0.99$, intercept $=0.001)$. The least agreement ( $\sim 5 \%$ average spread) between two ML-based approaches was observed in HCP $(r=0.95$, slope $=1.05$, intercept $=0.121)$. Intermediate results were observed in the NTR dataset $(r=0.98$, slope $=0.98$, intercept $=0.055)$. Hence, we averaged the heritability values produced by the two ML methods to create a "ground truth" reference for the two approximation methods.

The heritability estimates provided by the approximation approaches were more variable among three cohorts (Figure 2). The FPHI showed better accuracy in variance in slopes $(\beta=0.97-1.04)$ and intercepts $(\alpha=0.01-0.26)$ vs. APACE ( $\beta=0.61-0.73$ and intercepts $\alpha=-0.07-0.34$; Figure 2$)$. Both FPNI and APACE showed a modest negative bias. The highest bias was seen for the HCP cohort $(-0.08$ and -0.04 for APACE and FPNI, respectively). The bias in NTR and BrainSCALE cohorts was small $(-0.01$ and -0.02$)$. The spread for FPHI was about half that for APACE (6\% vs. $12 \%$ for $\mathrm{FPHI}$ and APACE, respectively).

\section{Heritability Analyses-Normalized Diffusion Data}

Next, heritability estimates were calculated on the residual data after inverse normal transformation (Figure 3). Trait normalization improved agreement among the ML-based methods $(r=0.96-0.99$, slope $=0.99-1.00$, intercept $=0.00-0.02$; Figure 3). 


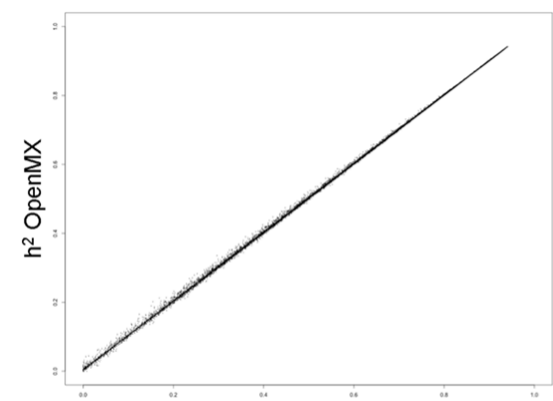

$h^{2}$ SOLAR-Eclipse

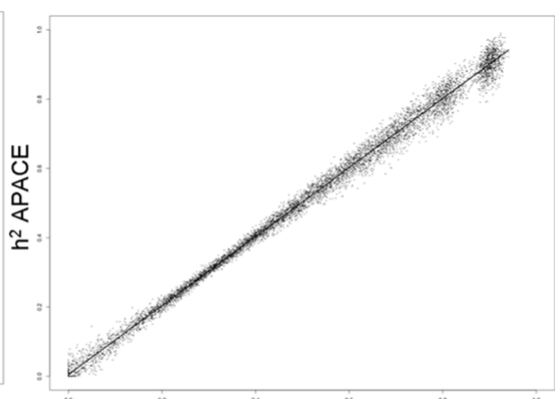

$h^{2}$ MLE

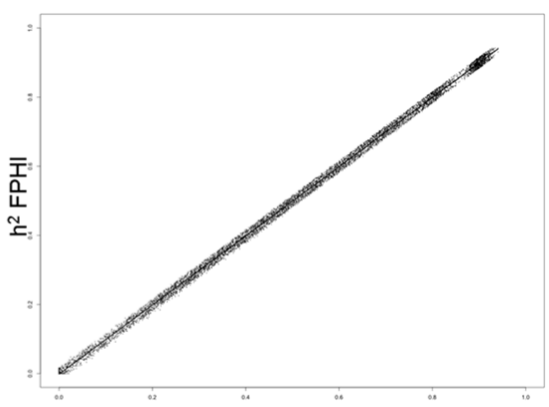

$\mathrm{h}^{2} \mathrm{MLE}$

FIGURE 1 | The scatter plot of heritability estimates for 10,000 simulated traits are shown for two ML-based approaches (left). Heritability estimates by two approximation approaches: accelerated permutation inference for ACE (APACE; center) and fast permutation heritability inference (FPHI; right) were plotted vs. the average maximum likelihood estimation (MLE) based values.

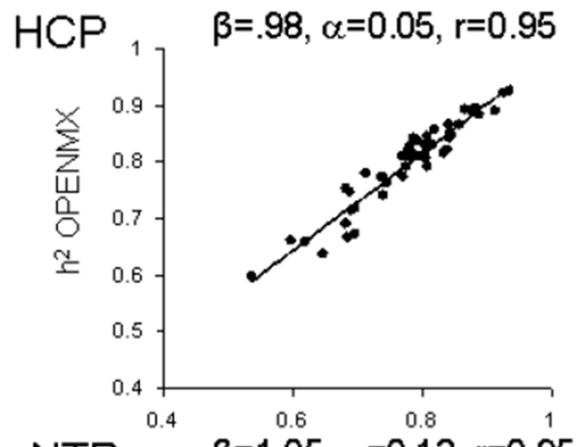

NTR $\quad \beta=1.05, \alpha=0.12, r=0.95$

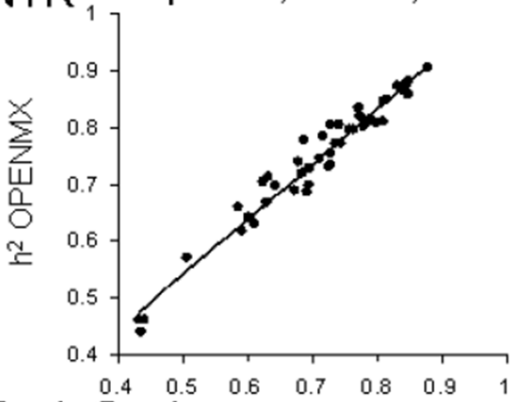

Brainscale

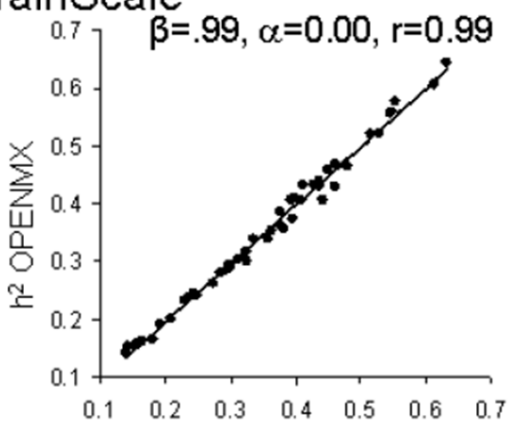

$h^{2}$ SOLAR-Eclipse
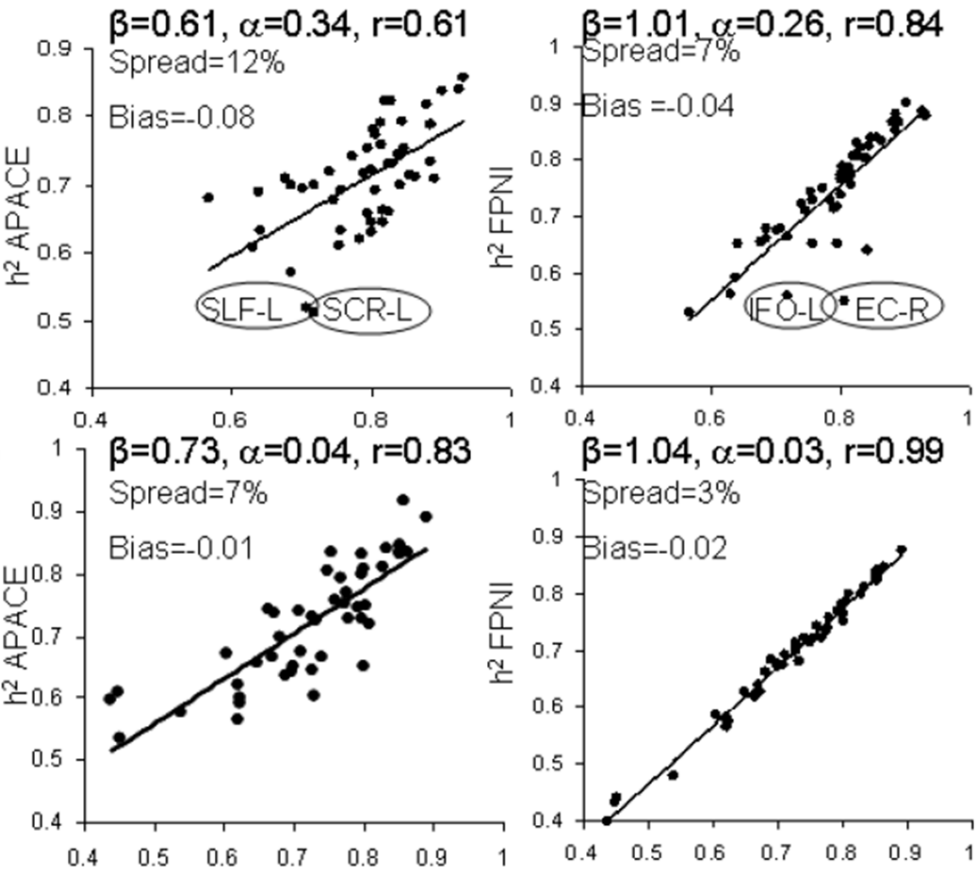

$\beta=1.04, \alpha=0.03, r=0.99$
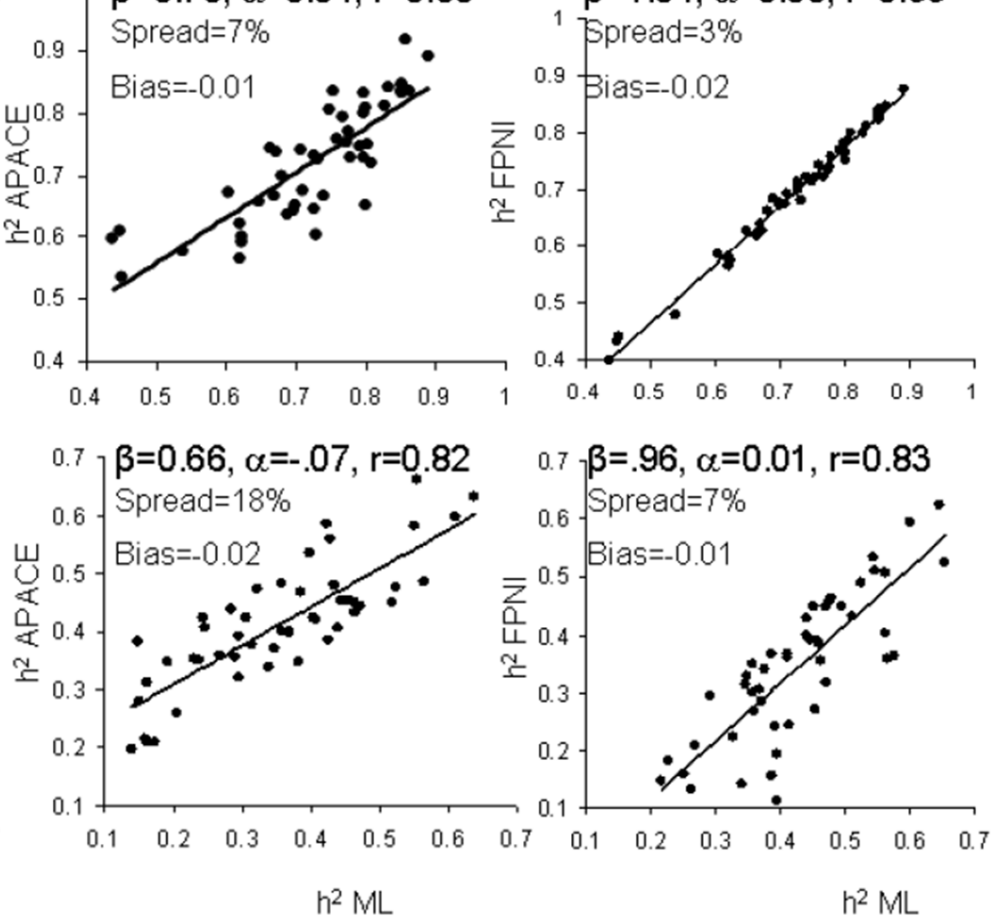

FIGURE 2 | The scatter plot of heritability estimates for 49-regional fractional anisotropy (FA) values calculated by the enhancing neuro imaging genetics analysis (ENIGMA)-diffusion tensor imaging (DTI) pipeline. Heritability estimates for two approximation approaches were plotted vs. the average estimate obtained for two ML-based methods: SOLAR-Eclipse and OpenMX. The lines represent linear regression fit vs. ML-based estimates with slope ( $\beta$ ), intercept $(\alpha)$ and Pearson correlation values (r). 

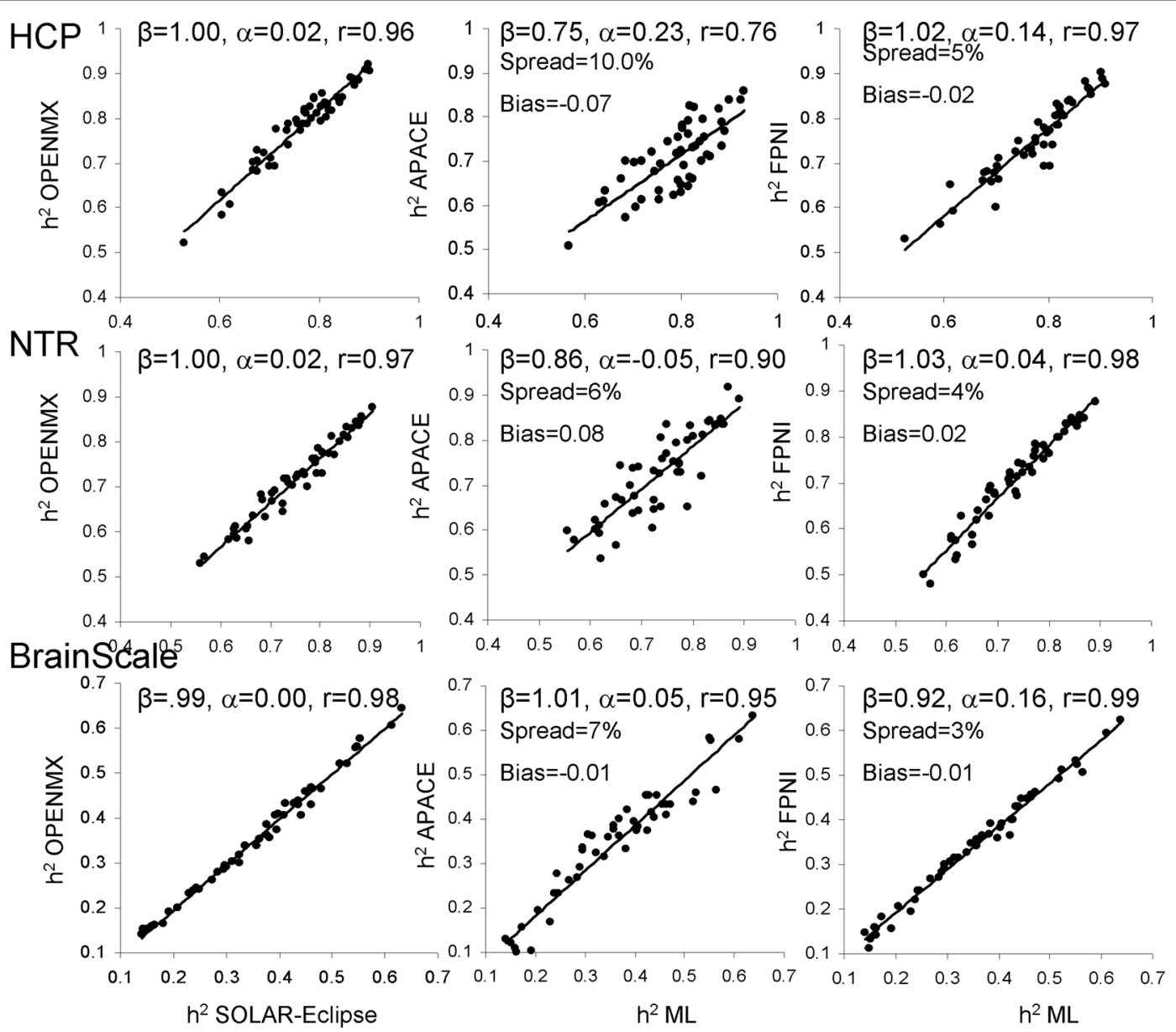

FIGURE 3 | The scatter plot of heritability estimates for 49-regional FA values calculated by ENIGMA-DTI pipeline and then normalized using the trait normalization function in SOLAR-Eclipse. Heritability estimates for two approximation approaches were plotted vs. the average estimate obtained for two ML-based methods: SOLAR-Eclipse and OpenMX. The lines represent linear regression fit vs. ML-based estimates with slope $(\beta)$, intercept $(\alpha)$ and Pearson correlation values $(r)$.

Trait normalization brought improvements in the agreement between the estimates by two approximation approaches and the average ML-based estimation (Figure 3). APACE method showed improvements in slope $(\beta=0.75-1.01)$, intercept $(\alpha=-0.05-0.23)$ and correlation coefficients $(r=0.76-0.95)$, in all three cohorts. For FPHI, the improvements were more subtle and were mainly observed as decrease in bias and spread. The bias for APACE increased for NTR cohort (from -0.01 to 0.08 ). Both approximation methods showed a 50\% improvement in the percentage spread vs. the average ML-based estimate, yet, the \% spread for FPHI remained about half that for APACE (4\% vs. $7.6 \%$ for FPHI and APACE, respectively).

\section{Analysis of the Disagreement}

We tested the normality of the distribution of the neuroimaging traits using the Shapiro-Wilk method, focusing on the HCP dataset because it had the largest number of subjects. We observed that four traits: the anterior limb of internal capsule-left (ALIC-L), uncinate fasciculus-right (UNC-R), external capsuleright (EC-R) and superior corona radiate-left (CR-L), failed the null hypothesis for normal distribution $(W>0.94, p<0.05$; Figure 2). However, there was no significant correlation between the deviation from normality or the heritability values for any of the four methods (all $r<0.20$, all $p>0.4$ ). Furthermore, some traits that visibly contributed to dispersion of heritability values, for example the inferior fronto-occipital tract-left (IFO-L) and superior corona-radiata-right (SCR-L; Figure 2), passed the Shapiro-Wilk test $(p>0.10)$. The histograms for SLF-L and SCR-L showed only modest kurtosis (kurtosis $=-0.2$ and 0.15 for SLF-L and SCR-L), but visibly varied from the normal distribution (Figure 4). The histograms for IFO-L varied visibly from a normal distribution despite having low kurtosis (0.13), while EC-R had high kurtosis (7.7; Figure 4).

\section{DISCUSSION}

We conducted a careful evaluation of four quantitative genetic approaches used by imaging genetic studies to measure heritability-the proportion of variance attributable to the additive genetic factors. Two of the methods (SOLAR-Eclipse 

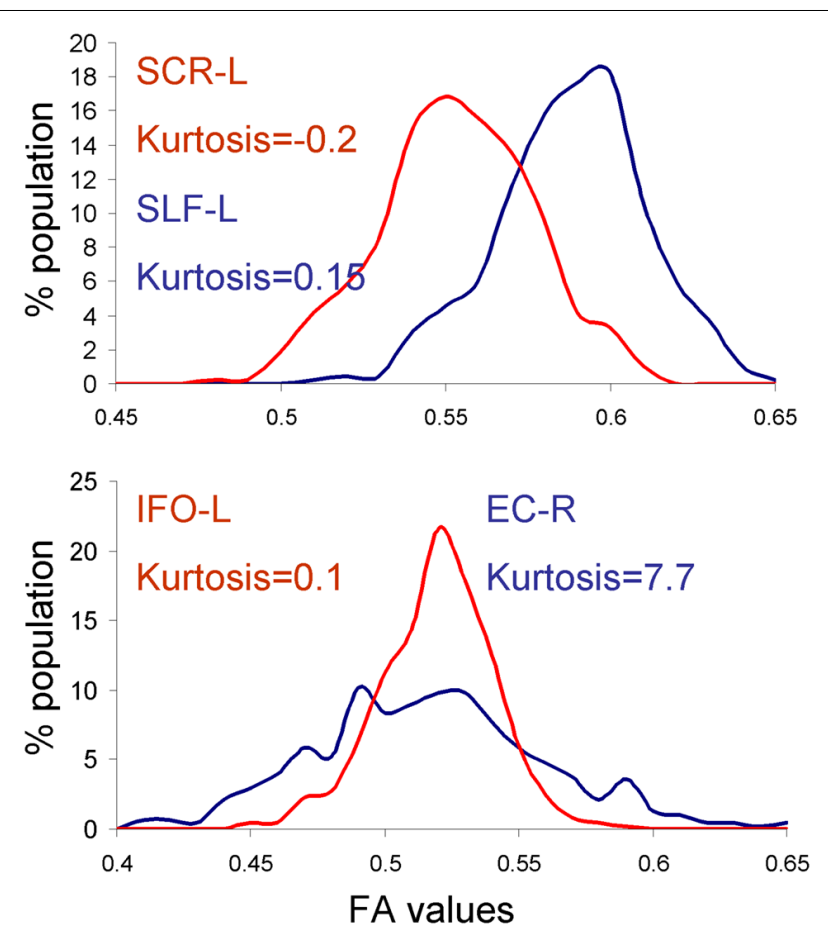

FIGURE 4 | Histograms for the dataset that showed reduced heritability estimates for fast vs. MLE based heritability estimation approaches. APACE showed reduced heritability estimates in superior corona-radiata-right (SCR-L) and SLF-L tracts in the human connectome project (HCP) cohort due to deviations from normal distribution (top panel). FPHI showed reduced heritability estimates in the external capsule-right (EC-R) and inferior fronto-occipital tract-left (IFO-L) tracts in the HCP cohort due to the high kurtosis and non-Gaussian shape of the histograms for EC-R and IFO-L, respectively.

and OpenMX) used an iterative MLE approach. Two methods (APCE and FPNI) were developed specifically to accelerate (by $10^{3-6}$ ) voxel-wise imaging genetics analyses using fast approximation approaches. We performed the evaluation in a simulated dataset and imaging data from three independent datasets. In the simulated data, we observed an excellent agreement between all heritability estimate approaches. The two MLE approaches accurately replicated the expected heritability values, with the unity slope and near zero intercept and measurement bias. The two approximation techniques likewise showed excellent agreement in the simulated data, with only slight spread (2.2\% and 3.7\% for FPNI and APACE, respectively). In neuroimaging data, the two MLE approaches produced consistent estimates of heritability for all cohorts. We used the average MLE as the reference measures for approximation techniques because the true additive genetic contribution is unknown (Parisi et al., 2014). In the neuroimaging data, the approximation methods showed deviations from MLE values that varied by the dataset and method. The approximation methods showed the best consistency for NTR and the lowest consistency in the HCP data. Post hoc analyses attempted to identify the sources of the dispersion based on the underlying distribution in imaging data. The heritability values were not significantly correlated with Shapiro-Wilk's $W$-value for any method or dataset (all $r<0.2$ ). However, the traits with high dispersion in heritability estimates did show deviations from normality in the underlying dataset. The heritability estimates produced by the FPHI approach were generally closest to that produced by MLE estimates. The agreement among all methods was significantly improved following data normalization approach that ensured normality for quantitative traits. This data normalization approach is now available as a part of SOLAR-Eclipse distribution.

Imaging genetics is a field that combines imaging and genetics - the two disciplines that have greatly advanced neuroscience in recent years. The replication challenges are not unique to this new field and require concerted efforts to address them. The main replication challenges that imaging genetics faces are the complexity of the methods and the low statistical power (Meyer-Lindenberg et al., 2008; Collins and Tabak, 2014). Genetic factors may explain a small proportion of variance that require a sample sizes that are challenging to collect in a single study $(N=1,000-100,000$; Stein et al., 2010, 2012; Thompson et al., 2014). Yet, imaging genetics approaches have many advantages that should help in overcoming this challenge. Modern MRI offers phenotypic measurements that provide more detailed and quantitative descriptions than disorder diagnostic status or clinical symptoms. Modern MRI phenotypes offer high precision and reproducibility with the inter-session, scan-rescan variability of many common imaging measurements in the range of 1\%-5\% (Agartz et al., 2001; Kim et al., 2005; Lerch and Evans, 2005; Kochunov and Duff Davis, 2009; Acheson et al., 2017). Therefore, the solution to statistical power is meta-analyses that combine data across multiple studies.

ENIGMA, Cohorts for Heart and Aging Research in Genomic Epidemiology (CHARGE) and other multi-study initiatives aim to overcome the challenge of limited power by performing meta-analytical analyses. In these initiatives, phenotypic and genetic analyses are performed by individual sites and meta-analytical aggregation is used to derive the overall estimates of genetic effects. The main challenge in this approach is overcoming the diversity and complexity of analytical and statistical approaches that may lead to variance in phenotype extractions and estimation of effect sizes (Meyer-Lindenberg et al., 2008; Collins and Tabak, 2014). This complexity exists on both imaging and genetic sides where the difference in analysis software and even versions of software may lead to varying results (Gronenschild et al., 2012). On the phenotype extraction side, ENIGMA provides the standardized pipeline for extraction of homogenized neuroimaging phenotypes across the sites (Jahanshad et al., 2013). Here, we demonstrate the need of homogenized treatments of the traits to avoid erroneous variances at the meta-analytical state.

In our evaluations, we observed excellent agreement between estimates produced by the two MLE-based approaches that were the corner stone of imaging genetic research in the past. The main disadvantage of MLE approaches is the long calculation times associated with the iterative maximization of the likelihood. In imaging genetic studies, up to a million voxelbased imaging traits may be analyzed (Stein et al., 2011), making MLE approaches less practical. Voxel-wise analyses require a 
permutation-based correction for multiple comparisons because standard multiple comparison approaches are deemed to be too conservative for voxel-wise traits (Nichols and Hayasaka, 2003). Therefore, there is a need for fast and accurate methods to estimate genetic variance where the calculations can be repeated with $10^{5-6}$ permutations to derive cluster-based significance on the voxel-wise levels. We measured the performance of two such methods (APACE and FPNI) that use approximation to obtain fast inference of genetic variance.

APACE and FPNI use data transformation and approximation fits to accelerate the calculation of genetic parameters. APACE uses a squared difference in phenotype values between pairs of related and unrelated subjects to derive the fraction of variance contributable to the additive genetic variance. This approach is appropriate for twins and siblings pedigree. FPNI uses the eigenvalue decomposition followed by a single step approximation to calculate genetic variance in pedigrees of any complexities. The approximation approaches demonstrated an excellent performance in the simulated dataset where the trait data was normally distributed. However, their performance in the imaging data was less uniform, likely due to sensitivity to noise and violations of the normality assumption.

The two MLE approaches appeared to provide more stable estimates of heritability in datasets with noise and the non-normally distributed traits, while these deviations had a greater impact on the heritability estimates produced by the approximation approaches. In the cases where the trait's distribution deviated from normality, the heritability values calculated by the approximation techniques deviated from those calculated by ML-based approaches. However, the correlation between heritability values and the deviation of normality (Shapiro-Wilk's W) was not significant. We explored four cases of visible outliers. Some traits (ALIC-L, UNC-R, EC-R and CR-L) failed assumptions for normality, but other outliers passed normality according to ShapiroWilk's test. We concluded that approximation approaches may be more sensitive to the noise and deviation from data normality and may produce biased heritability estimates even in traits whose distributions pass the standard tests for normality.

We found that the use of inverse normal transformation improved the agreement between $\mathrm{ML}$ and approximationbased approaches and resolved the outlier heritability estimates observed in uncorrected data. The inverse normal transformation did not alter the pattern of ML-based estimates: high correlation $(r>0.95)$ was observed for averaged ML-estimates before and after inverse normal transformation. Enforcing normality upon data reduced the dispersion in $\mathrm{h}^{2}$ values and improved the average spread for the approximation approaches. This was especially noticeable for FPNI approach where the correlations with ML-estimates became high $(r>0.97)$ for all cohorts.

\section{LIMITATION}

The ML estimations were used as the reference to compare the performance of approximation-based approaches in the simulated and imaging data. The two ML approaches produced convergent heritability estimates in both simulated and imaging datasets. However, this does not constitute the "ground truth" especially in imaging datasets where ML approaches may be biased despite convergence.

\section{CONCLUSION}

We have conducted a careful comparison of four heritability estimation methods for imaging data. Based on "groundtruth" simulations, four packages can produce lowbias, low-variance heritability estimates, with ML-based methods understandably performing slightly better than the approximation methods. In real data, the approximation methods exhibit more variability relative to the ML-based methods, but this variability was reduced with the use of a rank-based inverse normal transformation, suggesting that this may be an important tool to maximize intermethod reliability.

\section{ETHICS STATEMENT}

This study performed secondary data analyses in anonymized human subjects.

\section{AUTHOR CONTRIBUTIONS}

PK, BP, HG, BD, MR, XC, NJ, PT and TN designed experiment, performed analyses, and wrote the manuscript. All authors listed have made a substantial, direct and intellectual contribution to the work, and approved it for publication.

\section{FUNDING}

This study was supported by R01 EB015611 to PK, Foundation for the National Institutes of Health (NIH) BD2K grant, U54EB020403, R01 HD050735 to PT. This work was supported in part by a Consortium grant (U54 EB020403) from the NIH Institutes contributing to the Big Data to Knowledge (BD2K) Initiative, including the NIBIB and NCI. Data were provided by the HCP, WU-Minn Consortium (Principal Investigators: David Van Essen and Kamil Ugurbil; $1 \mathrm{U} 54 \mathrm{MH} 091657$ ) funded by the $16 \mathrm{NIH}$ Institutes and Centers that support the NIH Blueprint for Neuroscience Research; and by the McDonnell Center for Systems Neuroscience at Washington University. The NTR study (DvtE) was supported by the Netherlands Organization for Scientific Research [Medical Sciences (MW): grant no. 904-61-193; Social Sciences: grant no. 400-07-080; Social Sciences: grant no. 480-04-004]. The BrainSCALE study (HHP and DB) was supported by grants from the Dutch Organization for Scientific Research (NWO; 051.02.061) and 051.02.060. Computational support was provided by the NIH grant S10OD023696 to PK. 


\section{REFERENCES}

Acheson, A., Wijtenburg, S., Rowland, L., Winkler, A., Mathias, C. W., Hong, L., et al. (2017). Reproducibility of tract-based white matter microstructural measures using the ENIGMA-DTI protocol. Genes Brain Behav. 7:e0615. doi: 10.1002/brb3.615

Agartz, I., Okuguwa, G., Nordström, M., Greitz, D., Magnotta, V., and Sedvall, G. (2001). Reliability and reproducibility of brain tissue volumetry from segmented MR scans. Eur. Arch. Psychiatry Clin. Neurosci. 251, 255-261. doi: 10.1016/s1053-8119(01)92358-4

Alba-Ferrara, L. M., and de Erausquin, G. A. (2013). What does anisotropy measure? Insights from increased and decreased anisotropy in selective fiber tracts in schizophrenia. Front. Integr. Neurosci. 7:9. doi: 10.3389/fnint.2013.00009

Almasy, L., and Blangero, J. (1998). Multipoint quantitative-trait linkage analysis in general pedigrees. Am. J. Hum. Genet. 62, 1198-1211. doi: 10.1086/301844

Amos, C. I. (1994). Robust variance-components approach for assessing genetic linkage in pedigrees. Am. J. Hum. Genet. 54, 535-543.

Barysheva, M., Jahanshad, N., Foland-Ross, L., Altshuler, L. L., and Thompson, P. M. (2012). White matter microstructural abnormalities in bipolar disorder: a whole brain diffusion tensor imaging study. Neuroimage Clin. 2, 558-568. doi: 10.1016/j.nicl.2013.03.016

Basser, P. J., Mattiello, J., and LeBihan, D. (1994). MR diffusion tensor spectroscopy and imaging. Biophys. J. 66, 259-267. doi: 10.1016/S00063495(94)80775-1

Basser, P. J., and Pierpaoli, C. (1996). Microstructural and physiological features of tissues elucidated by quantitative-diffusion-tensor MRI. J. Magn. Reson. B 111, 209-219. doi: 10.1006/jmrb.1996.0086

Beasley, T. M., Erickson, S., and Allison, D. B. (2009). Rank-based inverse normal transformations are increasingly used, but are they merited? Behav. Genet. 39, 580-595. doi: 10.1007/s10519-009-9281-0

Blangero, J., Diego, V. P., Dyer, T. D., Almeida, M., Peralta, J., Kent, J. W. Jr., et al. (2013). A kernel of truth: statistical advances in polygenic variance component models for complex human pedigrees. Adv. Genet. 81, 1-31. doi: 10.1016/B9780-12-407677-8.00001-4

Blangero, J., Williams, J. T., and Almasy, L. (2001). Variance component methods for detecting complex trait loci. Adv. Genet. 42, 151-181. doi: 10.1016/s00652660(01)42021-9

Boker, S., Neale, M., Maes, H., Wilde, M., Spiegel, M., Brick, T., et al. (2011). OpenMx: an open source extended structural equation modeling framework. Psychometrika 76, 306-317. doi: 10.1007/s11336-010-9200-6

Bootsman, F., Brouwer, R. M., Schnack, H. G., Kemner, S. M., Hillegers, M. H. J., Sarkisyan, G., et al. (2016). A study of genetic and environmental contributions to structural brain changes over time in twins concordant and discordant for bipolar disorder. J. Psychiatr. Res. 79, 116-124. doi: 10.1016/j.jpsychires.2016. 04.011

Brouwer, R. M., Mandl, R. C., Peper, J. S., van Baal, G. C., Kahn, R. S., Boomsma, D. I., et al. (2010). Heritability of DTI and MTR in nine-yearold children. Neuroimage 53, 1085-1092. doi: 10.1016/j.neuroimage.2010. 03.017

Brouwer, R. M., Mandl, R. C., Schnack, H. G., van Soelen, I. L., van Baal, G. C., Peper, J. S., et al. (2012). White matter development in early puberty: a longitudinal volumetric and diffusion tensor imaging twin study. PLoS One 7:e32316. doi: 10.1371/journal.pone.0032316

Carballedo, A., Amico, F., Ugwu, I., Fagan, A. J., Fahey, C., Morris, D., et al. (2012). Reduced fractional anisotropy in the uncinate fasciculus in patients with major depression carrying the met-allele of the Val66Met brain-derived neurotrophic factor genotype. Am. J. Med. Genet. B Neuropsychiatr. Genet. 159B, 537-548. doi: 10.1002/ajmg.b.32060

Chen, X. (2014). Accelerated Estimation and Inference for Heritability of fMRI Data. Dissertation for PhD thesis. University of Warwick.

Chen, X., Blokland, G. A., Strike, L., and Nichols, T. (2013). Voxelwise and clusterbased heritability inferences of fMRI data. Proceedings of the 19th Annual Meeting for the Organization for Human Brain Mapping, 16-20.

Clerx, L., Visser, P. J., Verhey, F., and Aalten, P. (2012). New MRI markers for Alzheimer's disease: a meta-analysis of diffusion tensor imaging and a comparison with medial temporal lobe measurements. J. Alzheimers Dis. 29, 405-429. doi: 10.3233/jad-2011-110797
Collins, F. S., and Tabak, L. A. (2014). Policy: NIH plans to enhance reproducibility. Nature 505, 612-613. doi: 10.1038/505612a

den Braber, A., van't Ent, D., Blokland, G. A., van Grootheest, D. S., Cath, D. C., Veltman, D. J., et al. (2008). An fMRI study in monozygotic twins discordant for obsessive-compulsive symptoms. Biol. Psychol. 79, 91-102. doi: 10.1016/j. biopsycho.2008.01.010

den Braber, A., van 't Ent, D., Boomsma, D. I., Cath, D. C., Veltman, D. J., Thompson, P. M., et al. (2011). White matter differences in monozygotic twins discordant or concordant for obsessive-compulsive symptoms: a combined diffusion tensor imaging/voxel-based morphometry study. Biol. Psychiatry 70, 969-977. doi: 10.1016/j.biopsych.2011.03.029

den Braber, A., van 't Ent, D., Cath, D. C., Veltman, D. J., Boomsma, D. I., and de Geus, E. J. (2012). Brain activation during response interference in twins discordant or concordant for obsessive compulsive symptoms. Twin Res. Hum. Genet. 15, 372-383. doi: 10.1017/thg.2012.2

Friedman, J. I., Tang, C., Carpenter, D., Buchsbaum, M., Schmeidler, J., Flanagan, L., et al. (2008). Diffusion tensor imaging findings in first-episode and chronic schizophrenia patients. Am. J. Psychiatry. 165, 1024-1032. doi: 10.1176/appi. ajp.2008.07101640

Flint, J., and Munafò, M. R. (2013). Candidate and non-candidate genes in behavior genetics. Curr. Opin. Neurobiol. 23, 57-61. doi: 10.1016/j.conb.2012. 07.005

Ganjgahi, H., Winkler, A. M., Glahn, D. C., Blangero, J., Kochunov, P., and Nichols, T. E. (2015). Fast and powerful heritability inference for family-based neuroimaging studies. Neuroimage 115, 256-268. doi: 10.1016/j.neuroimage. 2015.03.005

Geng, X., Prom-Wormley, E. C., Perez, J., Kubarych, T., Styner, M., Lin, W., et al. (2012). White matter heritability using diffusion tensor imaging in neonatal brains. Twin Res. Hum. Genet. 15, 336-350. doi: 10.1017/thg.2012.14

Grimes, L., and Harvey, W. R. (1980). Estimation of genetic variances and covariances using symmetric differences squared. J. Anim. Sci. 50, 634-644. doi: $10.2527 /$ jas $1980.504634 \mathrm{x}$

Gronenschild, E. H., Habets, P., Jacobs, H. I., Mengelers, R., Rozendaal, N van Os, J., et al. (2012). The effects of FreeSurfer version, workstation type, and Macintosh operating system version on anatomical volume and cortical thickness measurements. PLoS One 7:e38234. doi: 10.1371/journal pone. 0038234

Ioannidis, J. P. A. (2014). How to make more published research true. PLoS Med. 11:e1001747. doi: 10.1371/journal.pmed.1001747

Jahanshad, N., Kochunov, P., Sprooten, E., Mandl, R. C., Nichols, T. E., Almassy, L., et al. (2013). Multi-site genetic analysis of diffusion images and voxelwise heritability analysis: a pilot project of the ENIGMA-DTI working group. Neuroimage 81, 455-469. doi: 10.1016/j.neuroimage.2013. 04.061

Jahanshad, N., Lee, A. D., Barysheva, M., McMahon, K. L., de Zubicaray, G. I., Martin, N. G., et al. (2010). Genetic influences on brain asymmetry: a DTI study of 374 twins and siblings. Neuroimage 52, 455-469. doi: 10.1016/j.neuroimage. 2010.04.236

Kim, J. S., Singh, V., Lee, J. K., Lerch, J., Ad-Dab'bagh, Y., MacDonald, D., et al. (2005). Automated 3-D extraction and evaluation of the inner and outer cortical surfaces using a Laplacian map and partial volume effect classification. Neuroimage 27, 210-221. doi: 10.1016/j.neuroimage.2005.03.036

Kochunov, P., Coyle, T., Rowland, L., Jahanshad, N., Thompson, P., Kelly, S., et al. (2017). White matter and core cognitive deficits in schizophrenia. JAMA Psychiatry 74, 958-966. doi: 10.1001/jamapsychiatry.2017.2228

Kochunov, P., and Duff Davis, M. (2009). Development of structural MR brain imaging protocols to study genetics and maturation. Methods 50, 136-146. doi: 10.1016/j.ymeth.2009.08.002

Kochunov, P., Glahn, D. C., Rowland, L. M., Olvera, R., Wincker, P., Yang, D., et al. (2013). Testing the hypothesis of accelerated cerebral white matter aging in schizophrenia and major depression. Biol. Psychiatry 73, 482-491. doi: 10.1016/j.biopsych.2012.10.002

Kochunov, P., Jahanshad, N., Marcus, D., Winkler, A., Sprooten, E., Nichols, T. E., et al. (2015). Heritability of fractional anisotropy in human white matter: a comparison of human connectome project and ENIGMA-DTI data. Neuroimage 111, 300-311. doi: 10.1016/j.neuroimage.2015.02.050

Kochunov, P., Jahanshad, N., Sprooten, E., Nichols, T. E., Mandl, R. C., Almasy, L., et al. (2014). Multi-site study of additive genetic effects on fractional anisotropy 
of cerebral white matter: comparing meta and megaanalytical approaches for data pooling. Neuroimage 95C, 136-150. doi: 10.1016/j.neuroimage.2014. 03.033

Kochunov, P., Lancaster, J., Thompson, P., Toga, A. W., Brewer, P., Hardies, J., et al. (2002). An optimized individual target brain in the Talairach coordinate system. Neuroimage 17, 922-927. doi: 10.1016/s1053-8119(02)91084-0

Kochunov, P., Thompson, P. M., Winkler, A., Morrissey, M., Fu, M., Coyle, T. R., et al. (2016). The common genetic influence over processing speed and white matter microstructure: evidence from the old order amish and human connectome projects. Neuroimage 125, 189-197. doi: 10.1016/j.neuroimage. 2015.10.050

Lerch, J. P., and Evans, A. C. (2005). Cortical thickness analysis examined through power analysis and a population simulation. Neuroimage 24, 163-173. doi: 10.1016/j.neuroimage.2004.07.045

Mandl, R. C., Rais, M., van Baal, G. C., van Haren, N. E., Cahn, W., Kahn, R. S., et al. (2013). Altered white matter connectivity in never-medicated patients with schizophrenia. Hum. Brain Mapp. 34, 2353-2365. doi: 10.1002/hbm. 22075

Marcus, D. S., Harms, M. P., Snyder, A. Z., Jenkinson, M., Wilson, J. A., Glasser, M. F., et al. (2013). Human connectome project informatics: quality control, database services, and data visualization. Neuroimage 80, 202-219. doi: 10.1016/j.neuroimage.2013.05.077

McGuire, S. A., Wijtenburg, S. A., Sherman, P. M., Rowland, L. M., Ryan, M., Sladky, J. H., et al. (2017). Reproducibility of quantitative structural and physiological MRI measurements. Brain Behav. 7:e00759. doi: 10.1002/ brb3.759

Meyer-Lindenberg, A., Nicodemus, K. K., Egan, M. F., Callicott, J. H., Mattay, V., and Weinberger, D. R. (2008). False positives in imaging genetics. Neuroimage 40, 655-661. doi: 10.1016/j.neuroimage.2007.11.058

Nazeri, A., Chakravarty, M. M., Felsky, D., Lobaugh, N. J., Rajji, T. K., Mulsant, B. H., et al. (2013). Alterations of superficial white matter in schizophrenia and relationship to cognitive performance. Neuropsychopharmacology 38, 1954-1962. doi: 10.1038/npp.2013.93

Nichols, T., and Hayasaka, S. (2003). Controlling the familywise error rate in functional neuroimaging: a comparative review. Stat. Methods Med. Res. 12, 419-446. doi: 10.1191/0962280203sm341ra

Parisi, F., Strino, F., Nadler, B., and Kluger, Y. (2014). Ranking and combining multiple predictors without labeled data. Proc. Natl. Acad. Sci. U S A 111, 1253-1258. doi: 10.1073/pnas.1219097111

Penke, L., Muñoz Maniega, S., Houlihan, L. M., Murray, C., Gow, A. J., Clayden, J. D., et al. (2010a). White matter integrity in the splenium of the corpus callosum is related to successful cognitive aging and partly mediates the protective effect of an ancestral polymorphism in ADRB2. Behav. Genet. 40, 146-156. doi: 10.1007/s10519-009-9318-4

Penke, L., Muñoz Maniega, S., Murray, C., Gow, A. J., Hernandez, M. C., Clayden, J. D., et al. (2010b). A general factor of brain white matter integrity predicts information processing speed in healthy older people. J. Neurosci. 30, 7569-7574. doi: 10.1523/JNEUROSCI.1553-10.2010

Pérez-Iglesias, R., Tordesillas-Gutiérrez, D., Barker, G. J., McGuire, P. K., Roiz-Santiañez, R., Mata, I., et al. (2010). White matter defects in first episode psychosis patients: a voxelwise analysis of diffusion tensor imaging. Neuroimage 49, 199-204. doi: 10.1016/j.neuroimage.2009.07.016

Shen, K.-K., Rose, S., Fripp, J., McMahon, K. L., de Zubicaray, G. I., Martin, N. G., et al. (2014). Investigating brain connectivity heritability in a twin study using diffusion imaging data. Neuroimage 100, 628-641. doi: 10.1016/j.neuroimage. 2014.06.041

Smith, S. M., Jenkinson, M., Johansen-Berg, H., Rueckert, D., Nichols, T. E., Mackay, C. E., et al. (2006). Tract-based spatial statistics: voxelwise analysis of multi-subject diffusion data. Neuroimage 31, 1487-1505. doi: 10.1016/j. neuroimage.2006.02.024

Sprooten, E., Sussmann, J. E., Clugston, A., Peel, A., McKirdy, J., Moorhead, T. W., et al. (2011). White matter integrity in individuals at high genetic risk of bipolar disorder. Biol. Psychiatry 70, 350-356. doi: 10.1016/j.biopsych.2011.01.021

Stein, J. L., Hua, X., Lee, S., Ho, A. J., Leow, A. D., Toga, A. W., et al. (2011). Voxelwise genome-wide association study (vGWAS). Neuroimage 53, 1160-1174. doi: 10.1016/j.neuroimage.2010.02.032

Stein, J. L., Hua, X., Morra, J. H., Lee, S., Hibar, D. P., Ho, A. J., et al. (2010). Genome-wide analysis reveals novel genes influencing temporal lobe structure with relevance to neurodegeneration in Alzheimer's disease. Neuroimage 51, 542-554. doi: 10.1016/j.neuroimage.2010.02.068

Stein, J. L., Medland, S. E., Vasquez, A. A., Hibar, D. P., Senstad, R. E., Winkler, A. M., et al. (2012). Identification of common variants associated with human hippocampal and intracranial volumes. Nat. Genet. 44, 552-561. doi: $10.1038 /$ ng.2250

Teipel, S. J., Wegrzyn, M., Meindl, T., Frisoni, G., Bokde, A. L., Fellgiebel, A., et al. (2012). Anatomical MRI and DTI in the diagnosis of Alzheimer's disease: a european multicenter study. J. Alzheimers Dis. 31, S33-S47. doi: 10.3233/jad2012-112118

Thomason, M. E., and Thompson, P. M. (2011). Diffusion imaging, white matter, and psychopathology. Annu. Rev. Clin. Psychol. 7, 63-85. doi: 10.1146/annurevclinpsy-032210-104507

Thompson, P. M., Martin, N. G., and Wright, M. J. (2010). Imaging genomics. Curr. Opin. Neurol. 23, 368-373. doi: 10.1097/WCO.0b013e32833b764c

Thompson, P. M., Stein, J. L., Medland, S. E., Hibar, D. P., Vasquez, A. A., Renteria, M. E., et al. (2014). The ENIGMA Consortium: large-scale collaborative analyses of neuroimaging and genetic data. Brain Imaging Behav. 8, 153-182. doi: 10.1007/s11682-013-9269-5

Ugurbil, K., Xu, J., Auerbach, E. J., Moeller, S., Vu, A. T., Duarte-Carvajalino, J. M., et al. (2013). Pushing spatial and temporal resolution for functional and diffusion MRI in the human connectome project. Neuroimage 80, 80-104. doi: 10.1016/j.neuroimage.2013.05.012

van Beijsterveldt, C. E., Groen-Blokhuis, M., Hottenga, J. J., Franic, S., Hudziak, J. J., Lamb, D., et al. (2013). The young netherlands twin register (YNTR): longitudinal twin and family studies in over 70,000 children. Twin Res. Hum. Genet. 16, 252-267. doi: 10.1017/thg.2012.118

Van Essen, D. C., Ugurbil, K., Auerbach, E., Barch, D., Behrens, T. E., Bucholz, R., et al. (2012). The human connectome project: a data acquisition perspective. Neuroimage 62, 2222-2231. doi: 10.1016/j.neuroimage.2012.02.018

van Soelen, I. L., Brouwer, R. M., Peper, J. S., van Leeuwen, M., Koenis, M. M., van Beijsterveldt, T. C., et al. (2012). Brain SCALE: brain structure and cognition: an adolescent longitudinal twin study into the genetic etiology of individual differences. Twin Res. Hum. Genet. 15, 453-467. doi: 10.1017/thg.2012.4

Conflict of Interest Statement: The authors declare that the research was conducted in the absence of any commercial or financial relationships that could be construed as a potential conflict of interest.

Copyright (c) 2019 Kochunov, Patel, Ganjgahi, Donohue, Ryan, Hong, Chen, Adhikari, Jahanshad, Thompson, Van't Ent, den Braber, de Geus, Brouwer, Boomsma, Hulshoff Pol, de Zubicaray, McMahon, Martin, Wright and Nichols. This is an open-access article distributed under the terms of the Creative Commons Attribution License (CC BY). The use, distribution or reproduction in other forums is permitted, provided the original author(s) and the copyright owner(s) are credited and that the original publication in this journal is cited, in accordance with accepted academic practice. No use, distribution or reproduction is permitted which does not comply with these terms. 